
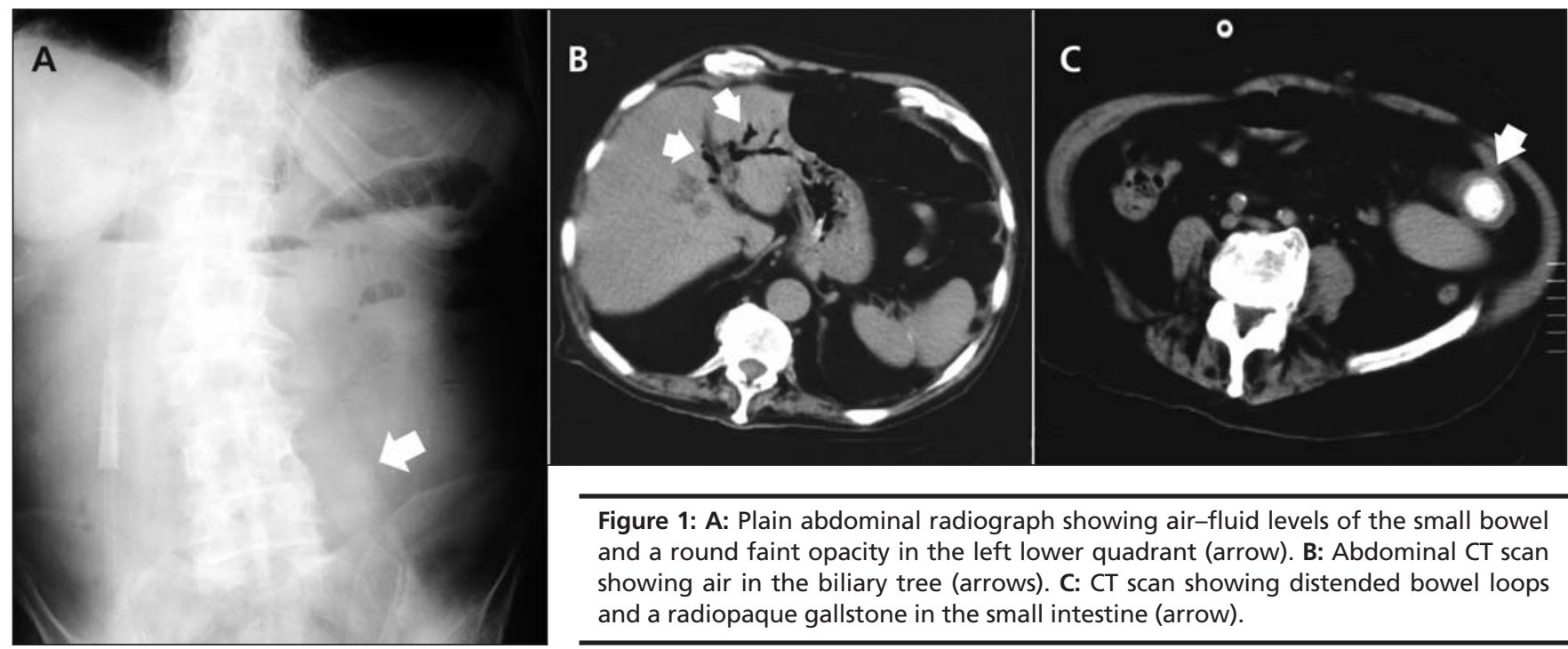

Figure 1: A: Plain abdominal radiograph showing air-fluid levels of the small bowel and a round faint opacity in the left lower quadrant (arrow). B: Abdominal CT scan showing air in the biliary tree (arrows). C: CT scan showing distended bowel loops and a radiopaque gallstone in the small intestine (arrow).

\title{
Gallstone ileus
}

$\mathrm{A}$ n 80-year-old man presented to the emergency department with a 1-day history of nausea, vomiting and epigastric pain. He reported having similar but milder episodes of abdominal pain in the past. The patient had no history of peptic ulcer disease or gallstones. On examination, there was mild epigastric tenderness and increased bowel sounds. A plain abdominal radiograph showed air-fluid levels in the small bowel and a faint round opacity (Figure 1A). An abdominal ultrasound showed air in the biliary tree (pneumobilia) but we had difficulty visualizing the gallbladder. The patient was admitted to hospital and received broad-spectrum antibiotics and intravenous fluids. The following day, he developed signs of an intestinal obstruction with absent bowel sounds. A computed tomography (CT) scan of the abdomen showed air in the biliary tree (Figure 1B) and a radiopaque gallstone in the small intestine (Figure 1C). The patient underwent an urgent enterolithotomy, and he had an uneventful recovery.

Gallstone ileus is a rare complication of cholecystitis that occurs in $0.5 \%$ of cases. ${ }^{1}$ It is usually associated with a biliary-enteric fistula, through which a gallstone passes to the bowel and causes a mechanical bowel obstruction. In patients aged 65 years and older, gallstone ileus accounts for $25 \%$ of cases of nonstrangulated small bowel obstructions. ${ }^{1}$ Most of these gallstones are larger than $2 \mathrm{~cm}$, and they become impacted in the ileum. ${ }^{2}$ The diagnosis of gallstone ileus is not always straightforward and is made preoperatively in about half of patients. Plain abdominal radiographs and abdominal CT scans are probably the most important diagnostic tests. Ultrasound images are often obscured by intestinal gas.

Clinical images are chosen because they are particularly intriguing, classic or dramatic. Submissions of clear, appropriately labelled high-resolution images must be accompanied by a figure caption and the patient's written consent for publication. A brief explanation (300 words maximum) of the educational significance of the images with minimal references is required.
Michael Samarkos MD MSc Virginia Makrygianni MD 5th Department of Internal Medicine Evagelismos Hospital Athens, Greece

\section{REFERENCES}

1. Reisner RM, Cohen JR. Gallstone ileus: a review of 1001 reported cases. Am Surg 1994;60:441-6.

2. Deitz DM, Standage BA, Pinson CW, et al. Improving the outcome in gallstone ileus. Am J Surg 1986;151:572-6. 